Journal Club

Editor's Note: These short, critical reviews of recent papers in the Journal, written exclusively by graduate students or postdoctoral fellows, are intended to summarize the important findings of the paper and provide additional insight and commentary. For more information on the format and purpose of the Journal Club, please see http://www.jneurosci.org/misc/ifa_features.shtml.

\title{
The Neural Correlates of Working Memory Impairment in Attention-Deficit/Hyperactivity Disorder
}

\author{
Hannah L. Pincham \\ Developmental Neuroscience Unit, Anna Freud Centre, London NW3 5SU, United Kingdom \\ Review of Lenartowicz et al.
}

Attention-deficit/hyperactivity disorder (ADHD) is a neurodevelopmental disorder characterized by age-inappropriate difficulties in attention, hyperactivity, and/or impulsivity. Working memory (WM) deficits constitute the key impairment in ADHD (Martinussen et al., 2005). Given that ADHD impedes educational and social development, and that brainbased research has the potential to aid diagnosis and help individualize treatment, a thorough understanding of the neural basis of WM impairments in ADHD stands to improve outcomes in children with ADHD.

Numerous researchers agree that WM is impaired in ADHD. However, behavioral and neuro-physiological evidence remains inconclusive. This is likely due to the multifaceted nature of WM (which, at the very least, comprises encoding and storage component processes) and the clinically heterogeneous nature of ADHD populations (with variability in symptom type and severity, age of onset, psychiatric comorbidities, and underlying causes). Research that uses objective measures to separate the component processes of WM is therefore required. In a recent paper published in The Journal of Neuroscience, Lenartowicz et al. (2014) responded to

Received Feb. 4, 2014; revised March 8, 2014; accepted March 13, 2014.

Thanks to James Kelly and Clare Killikelly for useful comments on this commentary.

Correspondence should be addressed to Hannah L. Pincham, 12 Maresfield Gardens, London NW35AH,UK. E-mail: hannah.pincham@annafreud.org. DOI:10.1523/JNEUROSCI.0487-14.2014

Copyright $@ 2014$ the authors $\quad 0270-6474 / 14 / 345735-03 \$ 15.00 / 0$ this need by comparing performance on the Sternberg Spatial Working Memory task across ADHD and typically developing (TD) children (aged 7-14 years). The authors used electroencephalography (EEG) to measure brain activity as participants performed different phases of the task.

The Sternberg Spatial WM task requires participants to decide whether the location of a probe stimulus matches the location of any stimulus previously presented during an encoding period. A major advantage of this task is that it is separable into three analysis phases: Alerting (during presentation of an initial fixation cross), Encoding (during presentation of the tobe-remembered stimulus array), and Maintenance (during a blank interval that appears between encoding and the probe stimulus). Lenartowicz et al. (2014) additionally manipulated the attentional load of the to-be-remembered stimulus array in order to vary the difficulty of the task.

Lenartowicz et al.'s (2014) behavioral results replicated existing research by demonstrating WM impairments in the ADHD group: accuracy was reduced and reaction times were more variable in the patient sample (for review, see FriedmanHill et al., 2010). But Lenartowicz et al.'s (2014) study went further by using cortical source-level EEG dynamics to examine ADHD across three working memory component processes. Specifically, the authors examined P2 peak amplitudes, parieto-occipital alpha $(8-12 \mathrm{~Hz})$ oscilla- tions, and fronto-central theta $(4-7 \mathrm{~Hz})$ oscillations.

One of their major results was that, during the Alerting phase, peak P2 amplitude (an index of selective attention or the identification of task-relevant, meaningful stimuli) was reduced in ADHD participants. This finding could not be accounted for by group differences in visual perception, thereby suggesting that task vigilance was poorer in the patient group.

Independent component and timefrequency analyses were used to examine parieto-occipital alpha and frontocentral theta frequency bands because these bands have been respectively implicated in WM encoding and WM maintenance. During the Encoding phase, alpha eventrelated desynchronization (ERD) was reduced in ADHD participants compared with TD participants, and this reduction was more pronounced under conditions of low attentional load. Interestingly, the vigilance deficit observed during the Alerting phase did not appear to cause the encoding deficits because P2 alerting amplitudes did not correlate with alpha ERD, hence WM deficits in ADHD appear to consist of abnormalities in both alerting and encoding. Finally, compared with TD children, theta event-related synchronization (ERS) during maintenance was enhanced in young people with ADHD. However, theta ERS significantly correlated with alpha ERD during encoding. Lenartowicz et al. (2014) argue that this correlation indicates that neural activity 
during the maintenance deficit might be driven by the preceding encoding deficit. In other words, abnormal WM maintenance might reflect a mechanism to compensate for abnormal WM encoding. However, one might argue that it is difficult to infer neural compensation because the behavioral data were poorer for the ADHD group.

Perhaps the most exciting result to emerge in Lenartowicz et al.'s (2014) data, at least from a clinical perspective, was the statistical model that the authors developed to predict an individual's ADHD diagnosis from their EEG data. Using P2 alerting amplitudes, alpha encoding ERD, and theta maintenance ERS as predictors in a logistic regression, Lenartowicz et al. (2014) classified participants as "ADHD" or "TD" with $70 \%$ accuracy, and determined that the odds of a participant having ADHD were five times greater given a positive results of the logistic regression rather than negative result. Based on their behavioral and EEG findings, Lenartowicz et al. (2014) concluded that ADHD is characterized by deficits at the level of WM vigilance and encoding. Correspondingly, interventions for $\mathrm{ADHD}$ should do well to focus on these component processes.

How does the work by Lenartowicz et al. (2014) fit in with previous work on the neural bases of ADHD? Considering the EEG literature, Lenartowicz et al.'s (2014) results are consistent with a recent report that frontocentral theta is linked to reaction time variability and ADHD symptomatology (McLoughlin et al., 2014). Considering functional magnetic resonance imaging (fMRI), a recent $\mathrm{ADHD}$ meta-analysis has concluded that, neurobiologically, $\mathrm{ADHD}$ is best defined by reductions in frontal lobe activity (McCarthy et al., 2014). The meta-analysis specifically implicated abnormalities in the left medial frontal cortex during WM tasks. Interestingly, the middle frontal gyrus (a component of the medial frontal cortex) has been identified as the neural generator of frontocentral EEG theta activity in a WM task (Zhao et al., 2014). It is therefore possible that abnormal medial frontal cortex activity in fMRI studies and abnormal frontocentral theta in EEG studies reflect the same underlying neural impairment. An empirical confirmation of this possibility could potentially unite the fMRI and EEG literatures concerning executive function deficits in ADHD.

Source localization techniques would be required to reveal the generator of theta power in Lenartowicz et al.'s (2014) study.
Regardless of the exact source, however, Lenartowicz et al. (2014) found that theta abnormalities during maintenance were preceded by attenuated alpha ERD during encoding. Attention-related alpha band oscillations are strongest over the occipital lobe. Lenartowicz et al.'s (2014) findings might therefore encourage fMRI studies of ADHD to examine the multiple component processes of WM, and to consider whether abnormal occipital activity precedes frontal abnormalities. Indeed, neuroimaging researchers that restrict their focus to a single component process of WM, or use region-of-interest analysis techniques (that focus on the frontal cortex rather than the whole brain), may have missed important pieces of the ADHD puzzle.

Lenartowicz et al.'s (2014) study contains a number of methodological strengths that deserve explicit recognition. As previously mentioned, the task effectively separated component processes of WM, helping to reveal the locus of the WM deficit often observed in ADHD. Further, patients were recruited using reliable diagnostic clinical interviews and most commonly comorbid psychiatric conditions were excluded. Unfortunately, this does not occur often enough in child clinical neuroimaging studies, where successful participant recruitment remains a real concern. Finally, the scope and depth of the authors' EEG analyses is unparalleled in this field. Notwithstanding those benefits, it is also important to note potential limitations.

For example, the number of participants eligible for inclusion in the logistic regression is potentially limiting. Initially, 102 participants were recruited. Independent components analysis reduced the sample size to 80 . Participants were then excluded from the model if their EEG data did not produce midoccipital and midfrontal clusters (because data from these clusters were required as predictors in the model), further reducing the sample size to 64 . Although it is statistically appropriate to only include participants who have valid data for each predictor, the fact that only $63 \%$ of children (64/102) could be included calls into question the diagnostic utility of the model outside the laboratory. Since brain-based diagnostic measures of ADHD are in their infancy, a restricted explanatory value is perhaps to be expected. Lenartowicz et al. (2014) acknowledge that further testing with larger sample sizes will be essential before neural measures can be used as diagnostic tools.
An additional consideration is Lenartowicz et al.'s (2014) inclusion of children with comorbid oppositional defiance disorder (ODD) in the ADHD group. Although ADHD and ODD are often comorbid, neuroimaging evidence suggests that these disorders may be underpinned by distinct neural substrates (Rubia, 2011; Zhu et al., 2014). It is therefore unclear whether Lenartowicz et al.'s (2014) findings are primarily driven by attention/hyperactivity/impulsivity (ADHD) dysfunctions or by disobedience/defiance (ODD). Though challenging, future research should aim to recruit pure ADHD cases. Interestingly, a neuroscientific hallmark of disinhibitory behavior (such as that observed in ODD) is reduced P3 amplitude (Iacono et al., 2002). Given that P2 and P3 amplitudes can temporally overlap in working memory tasks (Lefebvre et al., 2005), the body of literature demonstrating reduced $\mathrm{P} 3$ amplitude in young people with disinhibitory behavior might be relevant to Lenartowicz et al.'s (2014) finding of reduced P2 amplitude in children with ADHD.

Overall, Lenartowicz et al. (2014) clearly document the neural correlates of WM alerting, encoding, and maintenance in a sample of children with ADHD. In doing so, the authors highlight the importance of considering multiple WM component processes, and therefore help to disentangle the neural correlates underlying ADHD. Given Lenartowicz et al.'s (2014) thoughtful experimental design, careful participant recruitment, and rigorous statistical analysis, it is likely that their results will have implications for ADHD diagnosis, intervention evaluation, and treatment.

\section{References}

Friedman-Hill SR, Wagman MR, Gex SE, Pine DS, Leibenluft E, Ungerleider LG (2010) What does distractibility in ADHD reveal about mechanisms for top-down attentional control? Cognition 115:93-103. CrossRef Medline

Iacono WG, Carlson SR, Malone SM, McGue M (2002) P3 event-related potential amplitude and the risk for disinhibitory disorders in adolescent boys. Arch Gen Psychiatry 59:750757. CrossRef Medline

Lefebvre CD, Marchand Y, Eskes GA, Connolly JF (2005) Assessment of working memory abilities using an event-related brain potential (ERP)-compatible digit span backward task. Clin Neurophysiol 116:1665-1680. Medline

Lenartowicz A, Delorme A, Walshaw PD, Cho AL, Bilder RM, McGough JJ, McCracken JT, Makeig S, Loo SK (2014) Electroencephalography correlates of spatial working memory deficits in attention-deficit/hyperactivity 
disorder: vigilance, encoding, and maintenance. J Neurosci 34:1171-1182. CrossRef Medline

Martinussen R, Hayden J, Hogg-Johnson S, Tannock R (2005) A meta-analysis of working memory impairments in children with attention-deficit/hyperactivity disorder. J Am Acad Child Adolesc Psychiatry 44:377-384. CrossRef Medline

McCarthy H, Skokauskas N, Frodl T (2014) Identifying a consistent pattern of neural function in attention deficit hyperactivity disorder: a meta-analysis. Psychol Med 44:869880. CrossRef Medline
McLoughlin G, Palmer JA, Rijsdijk F, Makeig S (2014) Genetic overlap between evoked frontocentral theta-band phase variability, reaction time variability, and attention-deficit/ hyperactivity disorder symptoms in a twin study. Biol Psychiatry 75:238-247. CrossRef Medline

Rubia K (2011) “Cool” inferior frontostriatal dysfunction in attention-deficit/hyperactivity disorder versus "hot" ventromedial orbitofrontal-limbic dysfunction in conduct disorder: a review. Biol Psychiatry 69: e69-e87. CrossRef Medline

Zhao Y, Tang D, Hu L, Zhang L, Hitchman G,
Wang L, Chen A (2014) Concurrent working memory task decreases the Stroop interference effect as indexed by the decreased theta oscillations. Neuroscience 262:92-106. CrossRef Medline

Zhu Y, Ying K, Wang J, Su L, Chen J, Lin F, Cai D, Zhou M, Wu D, Guo C, Wang S (2014) Differences in functional activity between boys with pure oppositional defiant disorder and controls during a response inhibition task: a preliminary study. Brain Imaging Behav. Advanced online publication. Retrieved Jan. 4, 2014. doi:10.1007/ s11682-013-9275-7. CrossRef Medline 